\title{
A quantitative method to implement and to assess the single isocenter technique for breast cancer radiation therapy
}

\author{
Abdulhamid Chaikh',2, Sara Fayolle², Isabelle Gabelle-Flandin', Maud Marguet ${ }^{3}$, \\ Nicolas Docquiere1, Jean-Yves Giraud', Jacques Balosso ${ }^{1,2}$ \\ ${ }^{1}$ Department of Radiation Oncology and Medical Physics, Grenoble University Hospital, France \\ ${ }^{2}$ Université Grenoble-Alpes, Grenoble, France \\ ${ }^{3}$ Institute of Radiation Physics, Lausanne University Hospital, Grand-Pré 1, Lausanne, Switzerland
}

Received January 22, 2015; Revised May 26, 2015; Accepted May 28, 2015; Published Online August 11, 2015

\section{Original Article}

\begin{abstract}
Purpose: We propose a process of quality assurance to validate and implement the single isocenter technique for breast cancer radiotherapy. We evaluated the dosimetric and temporal gains using the single isocenter technique compared to classic source to skin distance (SSD) technique. Methods: 6 patients of breast cancer localization were studied. For each patient 2 treatment plans were generated. In plan 1 the dose was calculated using SSD technique. In plan 2 the dose was calculated using single isocenter technique. To implement the plan 2 a dosimetric analysis including monitor units (MU), isodose curves, cumulative and differential dose volume histograms cDVH, dDVH respectively, coverage index, conformity index for planning target volume were used. The measurements using a PMMA phantom consist of measuring point dose by an ionization chamber and 2D dose distributions using 2D diodes arrays. Wilcoxon signed rank and Spearman's tests were used to calculate $p$-value and correlation coefficient, respectively. Results: The single isocenter technique reduced the MU by average on $-30.1 \pm 13.6 \%,(p=0.03)$. We observed an improvement with statistical significance between the two techniques for the mean dose, minimum dose and volume receiving $95 \%$ of the prescribed dose without over-dosage. The analysis for $\mathrm{dDVH}$ showed that the dose distribution in the target volume calculated in the single isocenter technique is more homogeneous than the SSD technique. Wilcoxon test showed that the two treatment plans had the same quality $(p>0.05)$. The difference between calculated and measured dose was within $2.4 \pm 3.3 \%$ for absolute point dose and the percentage of points passing gamma criteria was on average $99.8 \pm 0.2 \%$. Conclusion: This method provides a quantitative evaluation and comparison of the two irradiation techniques for breast cancer and the consequences of the technical change on dose calculation.
\end{abstract}

Keywords: Breast Cancer; Single Isocenter; Radiotherapy

\section{Introduction}

The classic source to skin distance (SSD) technique for breast cancer treatment combines from 2 to 7 irradiation fields. An operator needs to enter the treatment room and change the patient position for the next treatment field. Hence, this technique is rather time consuming, but also introduces potential positioning errors that may lead to over or under-irradiation. ${ }^{1,2}$ This technique looks rather old fashion at the present time where up to date, linear accelerators allow complex volume irradiations from a single isocenter as for intensity-modulated radiation therapy (IMRT) and volumetric-modulated arc therapy (VMAT). Using a single isocentric technique (SIT) would be more relevant, all treatment fields would have the same isocenter, and the patient a unique position thus reducing the positioning variability and improving the reproducibility of treatment fields junctions. ${ }^{3,4,5,6}$
The switch from SSD to SIT is thus desirable but immediately raises the question of the equivalence of results and tolerance. Although, breast cancer is a frequent condition, it looks inappropriate to design randomized comparative trials having clinical issues as end point, to solve this question. According to the very fundamental principle of radiation therapy: Similar doses should yield similar effects. So a comparative analysis based on dosimetric comparative tools originated from quality assurance methods could help to solve this problem and provide to the radiation oncologists' clues solid enough to switch for SIT with or without adaptations of their prescriptions references.

In this study we will assess the impact of switching from the SSD technique to the SIT on dosimetry parameters including the total radiation time delivery (in terms of monitor units) 
in the case of breast cancer treatment. This has been achieved by appraising the discrepancies between these two techniques using statistical analysis of dosimetric data obtained from real clinical treatments.

\section{Methods and Materials}

\section{Clinical cases}

This study is based on 6 patients with breast cancer including 19 planning target volumes (PTV). These patients were chosen to cover the range of the different types of clinical situations, namely: Breast alone, breast with boost, breast and internal mammary chain (IMC) or IMC and supra-clavicular area. Table 1 shows the site locations, the number of PTV, the prescribed dose and the treatment fields for all patients. Clinicians delineated the anatomic borders of target structures and organs at risk (OAR).

\section{Planning CT-scans and contouring of structures}

The computed tomography (CT) images of each patient were loaded into the treatment planning system (TPS) Eclipse ${ }^{\circledR}$ (Varian, version 8.9). The virtual simulation for each patient was generated by a digitally reconstructed radiograph (DRR) and beam's eye view (BEV) information. Thus a treatment field was superimposed on the DRR to assess target localization. The target volume including the security margins and the organs at risk were jointly defined by the radiation oncologist and the medical physicist. In order to take into account the patient respiratory movements and possible positioning errors, a $7 \mathrm{~mm}$ margin was added to each of these clinical target volumes.

TABLE 1: Report of the site locations, the number of PTV, the prescribed dose and the treatment fields for all patients.

\begin{tabular}{|c|c|c|c|c|}
\hline Patients & PTV & $\begin{array}{c}\text { Prescribed dose / } \\
\text { dose per fraction [Gy] }\end{array}$ & Energy & Fields \\
\hline \multirow[t]{5}{*}{1} & Breast & $50.6 / 2.2$ & $6 \mathrm{MV}$ & 2 internals tangential \\
\hline & & & & 2 externals tangential \\
\hline & Supra clavicular & $50.6 / 2.2$ & $6 \mathrm{MV}$ & 1 interior \\
\hline & Boost & $10 / 2.5$ & $6 \mathrm{MV}$ & 2 internals tangential \\
\hline & & & $18 \mathrm{MV}$ & 2 externals tangential \\
\hline \multirow[t]{6}{*}{2} & Breast & $50.6 / 2.2$ & $6 \mathrm{MV}$ & 3 internals tangential \\
\hline & & & $18 \mathrm{MV}$ & 3 externals tangential \\
\hline & Supra clavicular & $48.3 / 2.1$ & $6 \mathrm{MV}$ & 2 interiors \\
\hline & & & $18 \mathrm{MV}$ & \\
\hline & Boost & $11.5 / 0.5$ & $18 \mathrm{MV}$ & 1 internals tangential \\
\hline & & & & 1 externals tangential \\
\hline \multirow[t]{5}{*}{3} & Breast & $50.6 / 2.2$ & $6 \mathrm{MV}$ & 2 internals tangential \\
\hline & & & & 2 externals tangential \\
\hline & Supra clavicular & $48.3 / 2.1$ & $6 \mathrm{MV}$ & 1 anterior \\
\hline & IMC & $46 / 2$ & $6 \mathrm{MV}$ & 1 anterior \\
\hline & & & $6 \mathrm{MeV}$ & 1 anterior \\
\hline \multirow[t]{4}{*}{4} & Breast & $50.6 / 2.2$ & $\overline{6} \overline{\mathrm{MV}}$ & 3 internals tangential \\
\hline & & & $18 \mathrm{MV}$ & 3 externals tangential \\
\hline & Supra clavicular & $50.6 / 2.2$ & $6 \mathrm{MV}$ & 2 anteriors \\
\hline & & & $18 \mathrm{MV}$ & \\
\hline \multirow[t]{7}{*}{5} & Breast & $50 / 2$ & $6 \mathrm{MV}$ & 3 internals tangential \\
\hline & & & $18 \mathrm{MV}$ & 3 externals tangential \\
\hline & Supra clavicular & $50 / 2$ & $6 \mathrm{MV}$ & 1 anterior \\
\hline & Boost & $10 / 2.5$ & $6 \mathrm{MV}$ & 3 internals tangential \\
\hline & & & $18 \mathrm{MV}$ & 3 externals tangential \\
\hline & IMC & $45 / 1.8$ & $6 \mathrm{MV}$ & 1 interior \\
\hline & & & $12 \mathrm{MeV}$ & 1 anterior \\
\hline \multirow[t]{7}{*}{6} & Breast & $50.6 / 2.2$ & $6 \mathrm{MV}$ & 3 internals tangential \\
\hline & & & $18 \mathrm{MV}$ & 3 externals tangential \\
\hline & Supra clavicular & 46.2 / 2.1 & $6 \mathrm{MV}$ & 1 anterior \\
\hline & Boost & $11.5 / 0.5$ & $6 \mathrm{MV}$ & 2 internals tangential \\
\hline & & & $18 \mathrm{MV}$ & 2 externals tangential \\
\hline & IMC & $46.2 / 1.8$ & $6 \mathrm{MV}$ & 1 anterior \\
\hline & & & $12 \mathrm{MeV}$ & 1 anterior \\
\hline
\end{tabular}




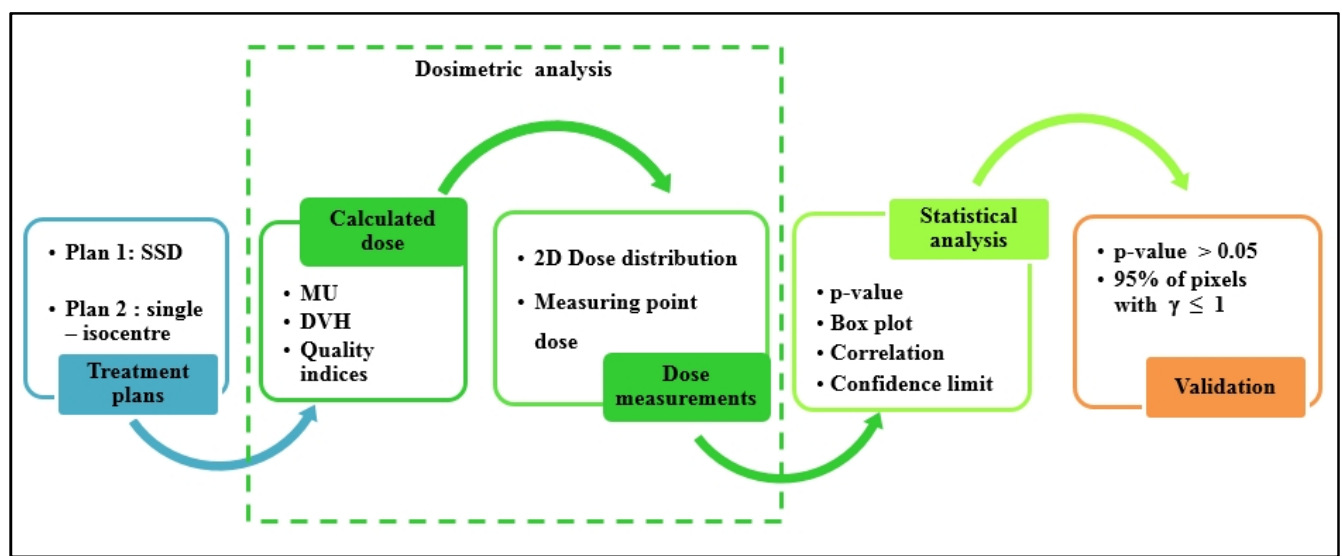

FIG. 1: Comprehensive quality assurance method including three successive steps to validate and implement the isocenter technique for breast cancer.
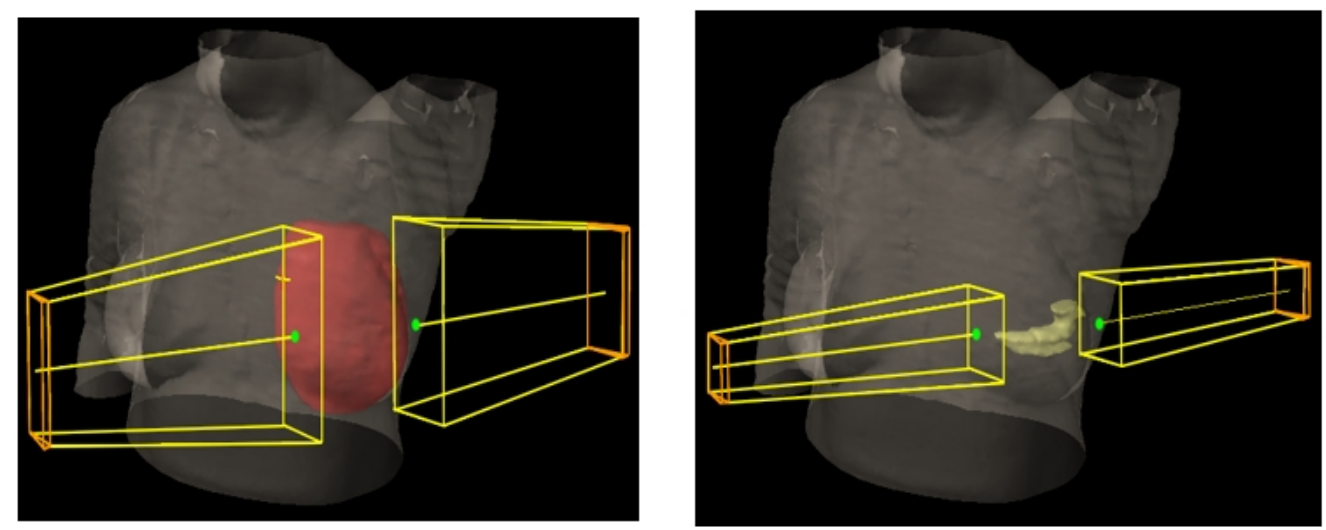

FIG. 2: Two opposed tangential fields to treat mammary gland using a wedge and an additional area such as boost. The left panel corresponds to the mammary gland area in orange, and right panel to boost area in yellow color.
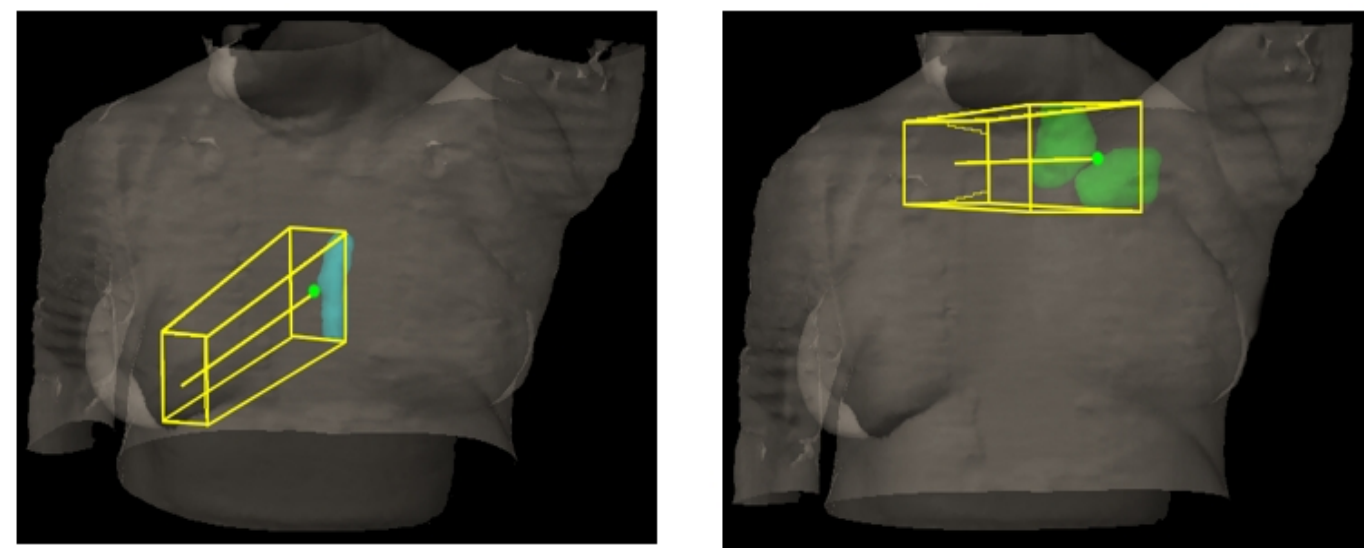

FIG. 3: One anterior photons and electrons beams to treat IMC area, and one anterior photons beam to treat supra-clavicular area. The left panel corresponds to IMC area in blue color, and right panel to supra-clavicular area in green color. Green point shows the beam entrance on the patient skin.

\section{Quality assurance method for implementing the single isocenter technique (SIT)}

The implementation and validation of the SIT consists of 3 successive steps including the generation of 2 treatment plans, the dosimetric and the statistical analysis, as shown in Figure 1.

\section{Treatment planning}

For each patient, two treatment plans were generated using Eclipse $^{\circledR}$ TPS:

- In plan 1 the dose was calculated using the SSD technique which was taken as the reference one, as shown in Figure 2 and 3. Patients were treated 
using the monitor units computed for this plan.

- In plan 2 the dose was calculated using the SIT.

The SSD technique was carried out as following: A CT was performed in treatment position, then the limits of the target volumes, namely the PTV, were drawn with the imaging tools of the Advantage Window ${ }^{\circledR}$ station (General Electric, USA). Crawling through the transverse images, the level of the thickest part of the breast was chosen to position the reference axis of the beams thus allowing to define the entrance point of both tangential beams axis. These points were marked on the patient's skin and pictures were taken. Using these data, the planification was completed using Eclipse $^{\circledR}$ TPS to make the dosimetric SSD plan and to calculate the beam parameters. At last, the dosimetric project was validated and verified respectively by the radiationoncologist and the medical physicist.

The dose was computed in plan 1 and plan 2 with the modified Batho correction density method in combination with the pencil beam convolution algorithm. To implement the beam configurations for SIT, the same CT images as for plan 1 were used with the same dose constrains for organs at risk as for plan 1. Figure 2 shows two opposed tangential fields to treat mammary gland and an additional boost using a wedge. As shown in the left and right panels, the mammary gland and boost were treated with 2 beams and presented in orange and yellow colors, respectively. Figure 3 shows one anterior photons and electrons beams to treat IMC area, and one anterior photons beam to treat supra-clavicular area. The left panel corresponds to IMC area in blue color, and right panel to supra-clavicular area in green color.

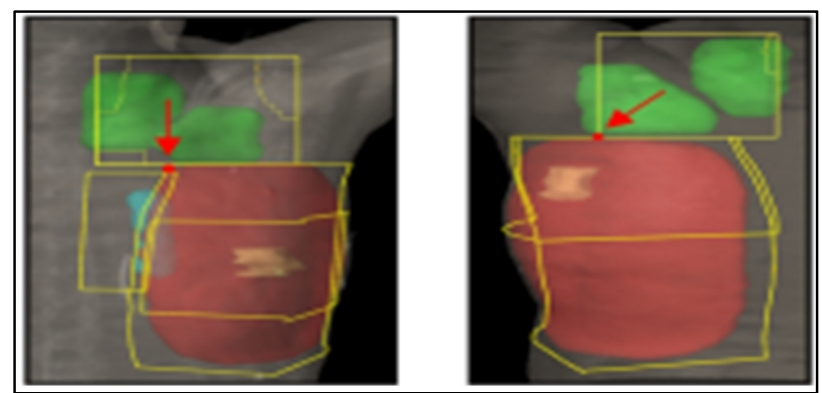

FIG. 4: Visualization of the position of the single isocenter, represented as a red point, on the projection of the irradiation fields placed on the skin of the patient. The unique isocenter was positioned on the patient skin. In the left panel, the unique isocenter positioned at the junction of the tangential to treat the breast, IMC and supra-clavicular areas represented in orange, blue and green respectively. In right panel, the unique isocenter was positioned on the patient skin at the field's junction for treat breast and supra-clavicular areas presented in orange and green colors respectively.

The isocenter was positioned according to the anatomical area to be irradiated. In the case of treatment of breast, IMC and supra-clavicular areas all together, the unique isocenter was positioned on the patient skin, at the junction of the tangential, IMC and supra-clavicular fields, as shown in Figure 4 (left panel). In the case of two areas treatment, the isocenter was positioned on the patient skin, at the field's junction, as shown in Figure 4 (right panel). The contoured target volumes on the computed tomography images allowed an optimal positioning of that isocenter. Figure 4 shows the position of the single isocenter, represented as a red point, on the projection of the radiation fields on the patient's skin. The geometrical parameters of each beam were then defined using the same type of field as with the SSD technique but without the wedges for they cannot be used with the single isocenter technique. Indeed, tangential fields are generated from a quarter of a beam and since the wedges are of limited size, they cannot fill up the entire tangential field area. Therefore, to obtain an homogeneous distribution of dose in the target volume, we used the "field-in-field" technique. ${ }^{7,8}$

With this technique the mammary gland is treated by two opposed tangential beams using the multi-leaf collimator (MLC) to protect areas where overdoses were observed. The shape of the MLC was defined using the BEV, and beams weight and energy were optimized as well as the normalization point position. Finally, we made sure that the collimator rotation was equal to zero degree for all fields, to avoid generating depth overlap and to make a perfect junction between the fields.

\section{Dosimetric analysis}

\section{Comparison of calculated dose}

Monitor units (MU)

The MU characterizes the irradiation duration. The calculation of the average and SD had been performed by summing MU for plan 1 and plan 2 for each patient and averaging with respect to the number of patients $(n=6)$.

\section{Isodose curves}

The $95 \%$ and $100 \%$ isodose curves encompass the PTV.

\section{Dose volume histogram (DVH)}

For each PTV the minimum dose (Dmin), mean dose (Dmean), maximum dose (Dmax), as well as the calculated dose to $95 \%$ of the PTV volume (D95) and the volume of PTV receiving $95 \%$ of the prescribed dose $\left(\mathrm{V}_{95}\right)$ were compared using cumulative DVH (cDVH). The differential DVH (dDVH) was used to compare the dose homogeneity inside the PTV using the standard deviation (S-index). Lastly we make sure that the dose tolerances of the three major organs at risk (OAR), i.e. lungs, spinal cord and heart, were met. For each of these organs and for each technique, the Dmean and Dmax were compared.

For each patient, to compare plan 2 with plan 1, the calculated dose in plan 1 was taken as the reference values. 
The difference in percentage was calculated as:

$$
\Delta \text { Dose }(\%)=\left(D_{\text {plan2 }}-D_{\text {plan1 }}\right) \times 100 / D_{\text {plan1 }}
$$

The positives values mean that the calculated dose using plan 2 (SIT) was higher than the calculated using plan 1 (SSD) and negative values would mean the opposite $\left(D_{\text {plan2 }}<D_{\text {plan1 }}\right)$.

\section{Quality indexes}

The coverage index (CI) and conformity index for planning target volume (CIP) were compared using the following equations ${ }^{9}$ :

$$
\mathrm{CI}=\frac{\mathrm{Imin}}{\mathrm{RI}}
$$

Where, Imin is the minimal isodose surrounding the target and $\mathrm{RI}$ is the reference isodose.

$$
\mathrm{CIP}=\frac{\mathrm{TV} 95 \%}{\mathrm{TV}}
$$

Where, TV59\% is target volume (TV) covered by the reference isodose $95 \%$.

\section{Comparison of calculated dose with measured dose}

In order to validate the single isocenter technique we make a consistent comparison for calculated dose with Eclipse ${ }^{\circledR}$ TPS and measured dose under the accelerator in the same condition. For this objective patient specific intensitymodulated radiation therapy quality assurance (IMRT-QA) methods have been used to validate the SIT. The QA for IMRT consists of measuring point doses and 2D dose distributions. The measurements were carried out according to AAPM (American Association of Physicist in Medicine) and ESTRO (European Society for Radiotherapy \& Oncology) guidelines. ${ }^{10,11}$ To compare the calculated dose with measured dose a CT-Scans of solid water slabs $(30 \times 30$ $\times 15 \mathrm{~cm}^{3}$ ) was used.

\section{Measuring point dose}

The pinpoint chamber 0.0125 cc was used. To measure the dose, the ballistic of plan 2 with the SIT was used. All parameters of the treatment plan were applied, a part of the gantry angle which was equal to 0 degree. The measurement was done using a Clinac $600^{\circledR}$ accelerator and the detectors placed at $5 \mathrm{~cm}$ depth in the phantom. The distance between the source and dosimeters was $100 \mathrm{~cm}$ along the beam axe. For each field the calculated dose was compared with the measured dose for three specific points. These points were selected in the flat domain of the dose profile calculated with the TPS. The difference between calculated dose $\left(D_{c}\right)$ and measured dose $\left(\mathrm{D}_{\mathrm{m}}\right)$ was calculated as follows:

$$
\Delta \mathrm{D} \%=\left(\mathrm{D}_{\mathrm{m}}-\mathrm{D}_{\mathrm{c}}\right) \times 100 / \mathrm{D}_{\mathrm{c}}
$$

The treatment plan was validated if 2 points upon 3 show less than $3 \%$ of difference.

\section{$2 D$ dose distributions}

The dose distribution showing a planar fluency map at $5 \mathrm{~cm}$ depth from the slab surface was calculated in the TPS and exported for comparison with the measured dose distribution. To compare the fluency map obtained from the TPS with dose fluency delivered by the accelerator, a 2-dimensional diode array (MapChek ${ }^{\circledR}$, Sun Nuclear, USA) was used. This detector is used for QA tests in IMRT. The dose measures were done taking account of the background correction factors and after the calibration procedure for the detector. The measurement of fluency map was carried out using a $0^{\circ}$ beam incidence to avoid the dependence of MapChek $^{\circledR}$ detector on beam incidence. Consistency of measurements and calculation was assessed using the $\gamma$ index with criteria of $(3 \%, 3 \mathrm{~mm})$. In this case the treatment plan was validated if $97 \%$ of the considered pixels were $<1$.

\section{Statistical analysis for dosimetric data}

Wilcoxon signed rank test was used to calculate the $p$-value. A bilateral statistical test was realized with an error $\alpha=5 \%$, corresponding to a $95 \%$ confidence interval. The dose difference is considered significant if $p<0.05$. Data are presented as average \pm standard deviation (SD). The box-and-whisker, boxplot, method was used to display the data set of 6 patients including all PTV $(n=19)$. Boxplot shows the minimum, maximum values as well as the $25^{\text {th }}$, $50^{\text {th }}$ (median) and $75^{\text {th }}$ percentile. The statistical correlation between calculated and measured dose was evaluated using Spearman's correlation coefficient. Correlation test can be used to measure the strength of the relationship between measured and calculated dose, i.e. estimate how much measured and calculated dose are related. ${ }^{12}$ The average difference between calculated and measured dose with SD were used to calculate the confidence limit CL:

$$
\mathrm{CL}=\mid \text { average } \mid+1.96 \times \mathrm{SD}
$$

For the gamma analyses, where perfect agreement produces a passing rate of $100 \%$, the confidence limit is defined as:

$$
\mathrm{CL}=(100-\text { average })+1.96 \times \mathrm{SD}
$$

where, average is the percentage of points passing the gamma criteria and SD is the standard deviation.

\section{Results}

\section{Dosimetric evaluation}

\section{Monitor Units}

The SIT reduced the MU by average on $(-30.1 \pm 13.6 \%)$ with a median of $-31.5 \%$ and $(p=0.03)$. This lower MU number is due in part to the fact that the SIT needs less numerous beams and in part to the collimator opening which is different for SIT.

\section{Isodose curves}

In the transversal plans, we observed that both $95 \%$ and 100 $\%$ isodose curves enclose the same fraction of PTV using the two techniques. 


\section{Dose volume histogram}

Figure 5 shows boxplot for $\mathrm{cDVH}$ indicating the minimum median and maximum values, the $25^{\text {th }}$ and $75^{\text {th }}$ percentile. The SIT reduced the under-irradiated volume D95\% by average on $(-7.36 \pm 23.3 \%)$ with a median of $2.5 \%$ and $(p=$ $0.01)$. In the case of $\mathrm{V} 95 \%$, we observed a mean difference of $1.3 \pm 14.4 \%$ with a median of $2 \%$ and $(\mathrm{p}=0.01)$. The analysis of the dDVH showed that the dose distribution within PTV was more homogeneous with the SIT than with the SSD one.
A mean difference for S-index of $(-7.2 \pm 45.3 \%$ with median of $-11.4 \%$ was observed. The Wilcoxon test supported the significance of this improvement $(p<0.001)$. For OAR the Table 2 shows dosimetric and statistical results obtained for lung, heart and spinal cord. For normal lung the dose constraints $\mathrm{V}_{20}<30 \%$ and $\mathrm{V}_{30}<20 \%$ were respected. The dose constraint for heart (maximum dose $\leq 35 \mathrm{~Gy}$ ) and spinal cord (maximum dose $\leq 45 \mathrm{~Gy}$ ) were respected in all plans.

TABLE 2: Dose difference for parameters deriving from dose volume histograms for organs at risks in all patients. $\Delta$ Dose $\%$ was calculated according to equation 1 , the negative sign means that SIT values are lower than SSD values. SD is the standard deviation. The $\mathrm{p}$-values were calculated using Wilcoxon signed rank test.

\begin{tabular}{|c|c|c|c|c|c|}
\hline \multicolumn{2}{|c|}{$\Delta$ Dose $\%$} & \multirow{2}{*}{$\begin{array}{c}\begin{array}{c}\text { Lung } \\
\text { homolateral }\end{array} \\
-2.1 \pm 4.3\end{array}$} & \multirow{2}{*}{$\begin{array}{c}\begin{array}{c}\text { Lung } \\
\text { contralateral }\end{array} \\
-8 \pm 32\end{array}$} & \multirow{2}{*}{$\frac{\text { Heart }}{3 \pm 16.4}$} & \multirow{2}{*}{$\begin{array}{c}\text { Spinal cord } \\
-13.3 \pm 34.9\end{array}$} \\
\hline Dmax & Average \pm SD & & & & \\
\hline \multirow{5}{*}{ Dmean } & Median & -2.9 & -10 & -2.6 & -13 \\
\hline & $p$-value & 0.4 & 0.2 & 0.7 & 0.3 \\
\hline & Average \pm SD & $-8.2 \pm 8.7$ & $-3.0 \pm 12.8$ & $17.6 \pm 23.1$ & $-2.4 \pm 9.9$ \\
\hline & Median & -6.9 & 0.6 & 25.9 & 0.9 \\
\hline & $p$-value & 0.1 & 0.3 & 0.2 & 1 \\
\hline
\end{tabular}

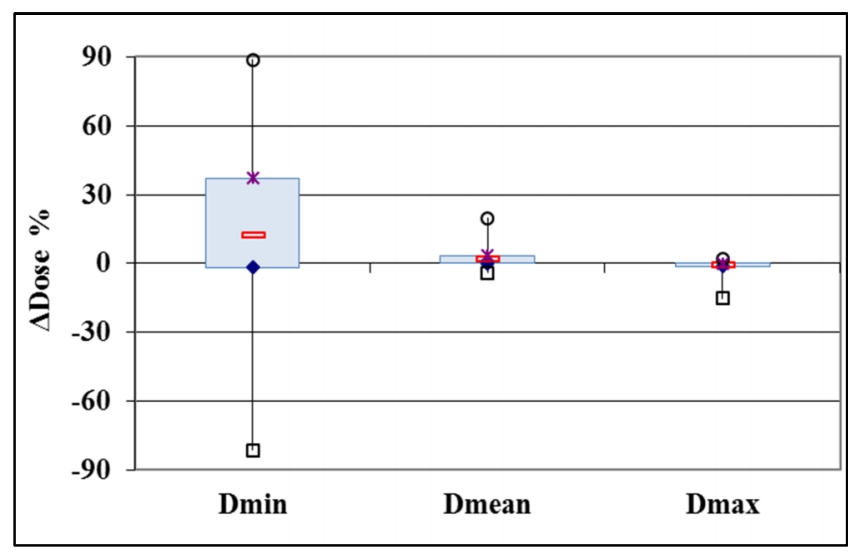

FIG. 5: Boxplot for dose difference obtained from cDVH parameters indicating the minimum, median in red line and maximum values, as well as the $25^{\text {th }}$ and $75^{\text {th }}$ percentiles. The median values of dose difference is close to zero for Dmean and Dmax.

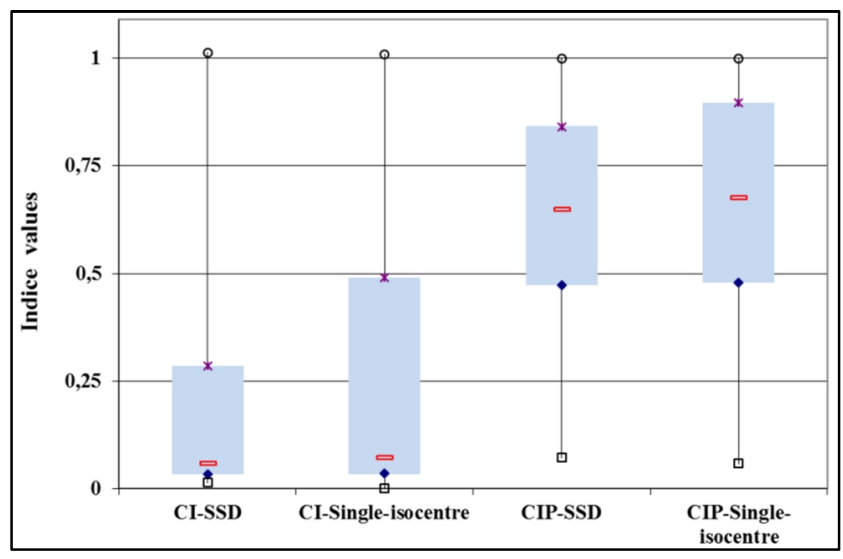

FIG. 6: Boxplot for quality indices indicating the minimum, median in red line and maximum values, as well as the $25^{\text {th }}$ and $75^{\text {th }}$ percentiles. The CI and CIP indices have the same median values for both techniques which have thus the same quality indices.

\section{Quality indices}

The comparison of CI and CIP showed that both techniques have the same quality. The averages of CI were $0.2 \pm 0.3$ and $0.3 \pm 0.4$ for plan 1 in SSD and plan 2 in SIT, respectively. The averages of CIP were $0.6 \pm 0.2$ and $0.7 \pm 0.3$ for plan 1 with SSD and plan 2 with SIT, respectively. Wilcoxon test showed that both techniques have the same dosimetric quality regarding CI and CIP indices with $p=0.22$ and $p=$ 0.27 respectively. Figure 6 represents the indices values using boxplot representation. It is clear that the CI has a median of 0.1 for both techniques. The CIP has a median of 0.6 and 0.7 for plan 1 and plan 2 respectively

\section{Discrete dose measurements}

For absolute point dose, the tolerance condition for 2 points among 3 having $3 \%$ or less of difference was respected. The difference between calculated and measured dose was within $2.4 \pm 3.3 \%$ and CL was 8.86\%, $p=0.02$ with strong correlation $r=0.99$. By using $\gamma$ criteria of $(3 \%, 3 \mathrm{~mm})$ for $2 \mathrm{D}$ dose analysis, we observed that the percentage of points passing the gamma criteria was on average $99.8 \pm 0.2 \%$ with $\mathrm{CL}=0.76 \%$.

\section{Discussion and Recommendations}

The irradiation duration in MU for the set of treatments is substantially shorter with SIT than with SSD one $(p<0.001)$. When analyzed by localizations, we were able to determine that the duration mainly decreased for the tangential beams treating the mammary gland. Since the time spent by the patient under the beam is critical for the economic efficiency of a radiotherapy department, this reduced number of MU is a valuable contribution to the advantages of the SIT. The $\mathrm{cDVH}$ and $\mathrm{dDVH}$ analysis for all patients showed a 
significant improvement $(p<0.05)$ using SIT in terms of maximum and mean doses, under-dosage, $\mathrm{V}_{95} \%$ and delivered dose homogeneity to the target volume. Again, identifying the PTV locations, we observed that the significant improvements were for the supra-clavicular and the mammary gland areas. No statistically significant differences were observed for quality indices, $(p>0.05)$.

The CI and CIP indices provide a qualitative comparison between plan 1 and plan 2 . The indexes quantify the degree of congruence between isodoses and prescribed dose. A care should be taken during interpretation the indices, since small changes in the dose distribution can dramatically change the conformity values. The s-index associated with dDVH provides a more reliable and accurate measure of dose homogeneity within the PTV.

Using single isocenter treatment plan, the calculated doses with TPS confirmed the results obtained for dose measurements. The AAPM suggested $4.5 \%$ for the CL between calculated and measured dose using ionization chamber. We observed a CL $>4.5 \%$ for all absolute dose measurements, but considering only the tolerance $2 / 3$ of points having $3 \%$ the CL was $<4.5 \%$.

This study has however several limitations. The main limitation is the number of patients. Nevertheless, Wilcoxon test can be used to calculate p-values for this small sample size. We were also confronted to a limit of the single isocenter technique when implementing the beam configurations. Indeed, the field size is limited since it used only the half of the maximum aperture size of the collimator jaws which is $20 \mathrm{~cm}$ on each side of the isocenter. This could be an issue to properly treat the lower limit of a large mammary gland.

To verify the implementation feasibility of the single isocenter technique, we recommend the following procedures:

- $\quad$ Localizing the normalization point of each field at $1 \mathrm{~cm}$ or more from the collimator blades to avoid the penumbra zones.

- Using an equivalent square field of, at least, $4 \times 4$ $\mathrm{cm}^{2}$ at the source-axis distance, smaller fields are not modeled.

- Using the field-in-field technique to obtain a homogeneous dose distribution in the PTV when IMRT is not used.

- Verifying the number of MU with independent computation.

- Verifying the enforcement of dose constraint for target volumes and OAR.

- $\quad$ QA by the measurement using IMRT-QA program to compare with the TPS calculated dose.

\section{Conclusion}

This paper presents a global approach for QA to implement the single isocenter method. This approach is based on dosimetric and statistical analysis methods. It enables the physicist and the radiation oncologists to be aware of treatment modifications associated with the change of SSD technique to single isocenter technique. We observed a reduction of irradiation duration and an improvement of the dose distribution homogeneity in the target volumes $(p<0.05)$ with the single isocenter technique. In addition no difference between treatment plan quality or healthy tissue and OAR preservation have been established $(p>0.05)$. The confidence limits of methods for measuring the point doses and 2D dose distributions were in accordance with AAPM and ESTRO guidelines. Therefore, the usage of the single isocenter technique could be considered in the radiation oncology department with full confidence.

\section{Conflict of interest}

The authors declare that they have no conflicts of interest. The authors alone are responsible for the content and writing of the paper.

\section{Acknowledgements}

The authors acknowledge the financial support of the French National Research Agency (ANR-11-TECS-018).

\section{References}

1. Klein EE, Taylor M, Michaletz-Lorentz M, et al. A mono isocentric technique for breast and regional nodal therapy using dual asymmetric jaws. Int J Radiat Oncol Biol Phys. 1994;28:753-60.

2. Idzes M, Holmberg O, Mijnheer BJ, Huizenga $H$. Effect of set-up uncertainties on the dose distribution in the match region of supraclavicular and tangential breast fields. Radiother Oncol. 1998;46: 91-8.

3. Marshall MG. Three-field isocentric breast irradiation using asymmetric jaws and a tilt board. Radiother Oncol. 1993; 28:228-32.

4. Rosenow UF, Valentine ES, Davis LW. A technique for treating local breast cancer using a single set-up point and asymmetric collimation. Int J Radiat Oncol Biol Phys. 1990; 19: 183-8.

5. Conte G, Nascimben O, Tureato G, et al. Three-field technique for breast evaluation using individualized shielding blocks. Int J Radiat Oncol Biol Phys.1988;14:1299-305.

6. Lu XQ Sullivan S, Eggleston T, et al. A three-field breast treatment technique with precise geometric matching using multileaf collimator-equipped lin- 
ear accelerators. Int J Radiat Oncol Biol Phys. 2003;55:1420-31.

7. Oozeer R, Aimard L, Alfonsi M, et al. Techniques single-isocentriques dans les cancers des VADS et les cancers du sein: mise en œuvre. Oncologie 2007;9:428-34.

8. Murthy KK, Sivakumar SS, Davis CA, et al. Optimization of dose distribution with multi-leaf collimator using field-in-field technique for parallel opposing tangential beams of breast cancers. J Med Phys. 2008;33:60-3.

9. Chaikh A, Giraud J, Balosso J. A method to quantify and assess the dosimetric and clinical impact re- sulting from the heterogeneity correction in radiotherapy for lung cancer. Int J Cancer Ther Oncol 2014; 2:020110.

10. Mijheer B, Georg D. Guidelines for the verification of IMRT. Brussels, Belgium: ESTRO; 2008.

11. Ezzell GA, Burmeister JW, Dogan N, et al. IMRT commissioning: multiple institution planning and dosimetry comparisons, a report from AAPM Task Group 119. Med Phys. 2009;36:5359-73.

12. Chaikh A, Giraud JY, Perrin E, et al. The choice of statistical methods for comparisons of dosimetric data in radiotherapy. Radiat Oncol. 2014; 9:205. 